\title{
Why Quantum of Quasars?
}

\author{
Cédric Foellmi ${ }^{* \dagger}$ \\ Laboratoire d'Astrophysique de Grenoble, 414 rue de la Piscine, 38400 Saint-Martin d'Hères \\ (France) \\ E-mail: cedric.foellmidobs.ujf-grenoble.fr
}

Why the Quantum of Quasars Workshop? Or more precisely: why reopening the questions of second-order photon correlations in astrophysics? The answer lies in the combination of three things. First, quantum optics made fantastic progresses since the original experiment by Hanbury Brown \& Twiss; progresses ignored so far in astrophysics. Second, the astrophysics community plans to built giant telescopes and telescope arrays. Third, there have been important technological breakthroughs in the field of ultra-fast low-light detectors.

More importantly, light carries much more information that its instantaneous frequency, intensity and polarization. For a scientific field aiming at exploring the Universe, and whose exploration tools are mostly based on light, it is stunning to realize that so far, astrophysics mostly remained at the first order of photon correlations, and furthermore extremely exciting to explore the possible links or applications of quantum light studies in astrophysics.

The slides of all talks are available at http: / / quantumof quasars . org.

Quantum of Quasars workshop - QQ09,

December 2-4, 2009

Grenoble, France

\footnotetext{
* Speaker.

†C.F. acknowledges support from Université Joseph Fourier Grenoble 1, the Laboratoire d'Astrophysique de Grenoble (LAOG) and the Rhône-Alpes région for their support to organize this workshop.
} 


\section{Quick History}

Fifty years ago, Hanbury Brown \& Twiss demonstrated the working principle of the intensity interferometry, and actually measured 32 stellar radii with the Narrabri Stellar Intensity Interferometer (see $1 ; 2 ; 3 ; 4 ; 5)$. For sensitivity reasons, this experiment has fallen into the darkness in the astrophysical community. On the other hand, what is now called quantum optics is a very active and fascinating field which still explore the HBT effect in laboratory to improve our understanding of the second-order correlations of photons. Today, quantum optics has solid theoretical grounds with the theory of optical coherence for which Roy Glauber earned the Physics Nobel Prize in 2005. But, astrophysics remained at the first-order of photon correlation, where light is a classical wave, governed by Maxwell equations.

The workshop aimed at asking the simple yet broad question: how can we make significant and conceptual progresses about our understanding of the physics of astronomical objects, and the way we observe the Universe by studying the results and the progresses of quantum optics about light and its quantum nature.

The pivotal element to the question "Why Quantum of Quasars Workshop?" is the fact that for measuring stellar radii, not only Hanbury Brown used second-order correlation function, but also, more or less explicitly, the following relationship:

$$
\left|g^{(1)}\right|^{2}=g^{(2)}-1
$$

This relationship is valid for chaotic light only. It means that as long as this is valid, it is possible to use $g^{(2)}$ to obtain a $g^{(1)}$ measurement (here an interferometric visibility). However, when it is broken, as it is the case in all non-thermal sources, or partially non-thermal sources, it opens an unknown territory for astrophysics. For a complete and detailed introduction on this central argument see (6).

\section{Open Questions}

The workshop aimed at mixing astrophysicists, quantum opticians and engineers in order to explore the links between fundamental optics, non-thermal astrophysical sources such as black holes and quasars, the non-linearity of light emission processes, and our ability to detect and make ultra-fast measurements with breakthrough detectors and giant telescopes. It was a very prospective workshop, and by many ways, significantly in advance for its time. What the Quantum of Quasars workshop revealed, as I expected, is how clear is the fact that we, astrophysicists, have the same vocabulary as quantum optics for everything related to interferometry.

The workshop was organized around the following questions:

- What value the function $g^{(2)}(t)$ can take in astrophysical sources? Is it observable with the Very Large Telescope? (See talk by Ivan Capraro, PoS ( 2009 ) 012)

- What means different $g^{(2)}(t)$ values about the emission process? (See talk by Hugues de Chatellus, POS (2009) 008). 
- What means a second-order spatial correlation value in general? For an astrophysical source? How coupled are the temporal and spatial coherence in astronomical observations? (See talk by Markus Schoeller, PoS (QQ09) 002).

- What modern intensity interferometers will do, be capable of, and what for? (See talk by David Kieda, PoS (Q209) 006).

- How profound is the Hanbury-Brown \& Twiss effect? With atoms? (See talk by Chris Westbrook, PoS ( Q209) 005).

- How can we achieve photon-counting detection at the "Heisenberg speed"? (See talk by Johan Rothman, PoS (QQ09) 009). On an Extremely Large Telescope?

- To what speed the HBT effect can be measured? How things become clearer, or different, at a much increased speed? (See talk by Emmanuel Rosencher, PoS (QQ09) 016).

- Can intricated photons be measured/detected? If yes, how? What meaningful measurement could be made with a Extremely Large Telescope at the highest speed? (See talk by Javier Gomez, PoS (2Q09) 015).

- Is there any interesting technique different from simple photon-counting for astrophysics? (See talk by François Reynaud, PoS (QQ09) 013).

- What first and second-order coherence can tell us on black hole objects? (See talk by Guy Pelletier, PoS (QQ09) 010).

- Can the degree of coherence be a new probe of (micro)quasars jets? Black holes? (See talk by Gilles Henri, PoS (Q209) 014).

- How coherence is affected by light-matter interactions? (See talk by Jean-Louis Le Gouët, POS ( QQ09) 007).

- Is there any mechanisms to break coherence? What about decoherence? (See talk by Brahim Lamine, POS (QQ09) 011).

- Finally, are there quantum light sources in the Universe? Around black holes? (There are already theoretical considerations about maximally entangled polarized photons via the Unruh effect.) Are they observable? See (6).

\section{The Future}

In the future, we will have 40-m class telescopes such as the E-ELT built by E.S.O. in Chile, giant telescope arrays such as the Cherenkov Telescope Array with which it will be possible to make intensity interferometry, and ultra-fast detectors allowing us to make efficient photon-counting with enough photons in the visible and infrared domains. Naturally, the questions related to secondorder photon correlations and quantum light will emerge in astrophysics in the coming years.

This workshop will be recognized as the pioneering one of the field. 


\section{References}

[1] R. H. Brown and R. Q. Twiss, Interferometry of the intensity fluctuations in light. i. basic theory: The correlation between photons in coherent beams of radiation, Proc. of the Royal Society of London. Series A 242 (Nov, 1957) 300.

[2] R. H. Brown and R. Q. Twiss, Interferometry of the intensity fluctuations in light ii. an experimental test of the theory for partially coherent light, Proc. of the Royal Society of London. Series A 243 (Jan, 1958) 291.

[3] R. H. Brown and R. Q. Twiss, Interferometry of the intensity fluctuations in light. iii. applications to astronomy, Proc. of the Royal Society of London. Series A 248 (Nov, 1958) 199.

[4] R. H. Brown and R. Q. Twiss, Interferometry of the intensity fluctuations in light. iv. a test of an intensity interferometer on sirius a, Proc. of the Royal Society of London. Series A $\mathbf{2 4 8}$ (Nov, 1958) 222.

[5] R. H. Brown, The intensity interferometer: Its application to astronomy, New York, Halsted Press (1974).

[6] C. Foellmi, Intensity interferometry and the second-order correlation function g(2) in astrophysics, A\&A $\mathbf{5 0 7}$ (Dec, 2009) 1719. 